\title{
Linear entropy and Bell inequalities
}

\author{
E. Santos* \\ Departamento de Física Moderna, Universidad de Cantabria, 39005 Santander, Spain \\ M. Ferrero ${ }^{\dagger}$ \\ Departamento de Física, Universidad de Oviedo, 33007 Oviedo, Spain
}

(Received 12 November 1999; published 19 July 2000)

\begin{abstract}
For mixed states of a pair of spin-1/2 particles, the positivity of the sum of the conditional linear entropies is a sufficient condition for the nonviolation of the Bell-CHSH (Clauser-Horne-Shimony-Holt) inequalities.
\end{abstract}

PACS number(s): 03.65.Bz, 89.70.+c

Entropy inequalities in clasical and quantum information have been studied since long ago [1], but recently they are receiving increasing attention [2] in view of the application to quantum computers and quantum communication. In recent times, information-theoretic Bell-type inequalities [3,2] have been derived, and a relation between the entropy inequality and the standard Bell-CHSH (Clauser-HorneShimony-Holt) [4] inequality has been established [5]. In this paper we prove, using the so-called linear entropy, that for mixed states of a pair of spin-1/2 particles, the positivity of the sum of the conditional entropies is a sufficient condition for the Bell-CHSH inequality.

The difference between classical and quantum information may be formalized in terms of the entropy. If $S_{12}$ is the entropy of the composite system and $S_{1}, S_{2}$ those of the subsystems, the classical statement that we cannot have more information about the whole than about each part may be represented by the inequality

$$
S_{2 / 1}+S_{1 / 2} \geqslant 0, \quad S_{i / j}=S_{12}-S_{j}, \quad j=1,2,
$$

where $S_{2 / 1}$ and $S_{1 / 2}$ are called conditional entropies. Actually, the stronger condition holds that each conditional entropy must be non-negative, but here we shall use only Eq. (1). The inequality is violated by quantum mechanics in some cases, for instance in the singlet spin state, where $S_{12}=0$, but both $S_{1}$ and $S_{2}$ are positive. In this paper we shall not use the standard von Neumann [6] entropy, but the more simple one defined by

$S_{12}=\operatorname{Tr}[\rho(1-\rho)]=1-\operatorname{Tr}\left(\rho^{2}\right), \quad S_{j}=1-\operatorname{Tr}\left(\rho_{j}^{2}\right), \quad j=1,2$,

where $\rho$ is the $4 \times 4$ density matrix representing the state of the two-particle system and $\rho_{1}\left(\rho_{2}\right)$ the $2 \times 2$ reduced density matrix of the first (second) particle. The classical counterpart of Eq. (2), called Tsallis entropy [7] of order 2, fulfills Eq. (1).

A relevant question is whether the violation of Eq. (1) is merely a theoretical feature derived from the definition of quantum entropy, or instead has empirical consequences.

\footnotetext{
*Electronic address: santose@besaya.unican.es

${ }^{\dagger}$ Electronic address: ferrero@pinon.ccu.uniovi.es
}

Horodecki et al. [5] proved that if both $S_{2 / 1} \geqslant 0$ and $S_{1 / 2}$ $\geqslant 0$, then no Bell inequality can be violated. Here we prove a stronger statement:

Theorem. For any state (mixed in general) of a system consisting of two spin-1/2 particles, the entropy inequality (1) is a sufficient condition for the nonviolation of the BellCHSH [4] inequalities.

Local realism implies constraints on the statistics of two widely separated systems. These constraints are generically known as Bell inequalities and arise from the assumption of the existence of an underlying joint probability for a set of measurable quantities. The CHSH Bell-type inequality applies to a pair of two-state systems and constrains the value of a linear combination of four correlation functions between the two systems. As is well known, the CHSH inequality can be violated by the statistical predictions of quantum mechanics.

In order to prove the theorem we consider two dichotomic observables, that is, Hermitian and traceless operators, $a_{1}$ and $b_{1}\left(a_{2}\right.$ and $\left.b_{2}\right)$, for the first (second) particle fulfilling the conditions

$$
a_{1}^{2}=b_{1}^{2}=a_{2}^{2}=b_{2}^{2}=1,
$$

and define the "Bell operator"' [8] by

$$
B=a_{1} a_{2}+a_{1} b_{2}+b_{1} a_{2}-b_{1} b_{2} .
$$

The density matrix $\rho$ fulfils the equalities

$$
\operatorname{Tr} \rho=1, \quad \operatorname{Tr}(\rho B)=\beta,
$$

$\beta$ being a real number in the interval $[-2 \sqrt{2}, 2 \sqrt{2}]$. The BellCHSH inequality is violated in the state $\rho$ if $|\beta|>2$. We shall prove (see below) that

$$
S_{1 / 2}+S_{2 / 1} \leqslant \frac{1}{2}\left[1-\frac{1}{4} \beta^{2}\right],
$$

which shows that all possible Bell-CHSH inequalities are fulfilled if the entropy inequality (1) holds true, which is the statement of the theorem.

In order to prove Eq. (6), we first define a different density matrix:

$$
\rho^{\prime}=\rho-\frac{1}{2} I_{1} \otimes \rho_{2}-\frac{1}{2} \rho_{1} \otimes I_{2}+\frac{1}{2} I
$$

where $I=\left(I_{1}, I_{2}\right)$ is the unit $4 \times 4(2 \times 2)$ matrix and $\otimes$ means tensor product. It is easy to see that if $\rho$ fulfills Eqs. (5) 
$\rho^{\prime}$ also fulfills it with the same $\beta$, but each reduced density matrix of $\rho^{\prime}$ is one-half the unit matrix. The relevant result is that $S_{1 / 2}+S_{2 / 1}$ is the same for $\rho$ as it is for $\rho^{\prime}$, which allows one to write it in terms of the entropy $S_{12}^{\prime}$ of $\rho^{\prime}$; that is,

$$
S_{1 / 2}+S_{2 / 1}=S_{1 / 2}^{\prime}+S_{2 / 1}^{\prime}=2 S_{12}^{\prime}-1 .
$$

The second Eq. (8), may be derived easily from Eqs. (1) and (2), taking into account that $\rho_{j}^{\prime}=\frac{1}{2} I_{j}(j=1,2)$. The proof of the first equality is as follows. From Eq. (7) we get

$$
\operatorname{Tr}\left(\rho^{\prime}-\frac{1}{2} I\right)^{2}=\operatorname{Tr}\left(\rho-\frac{1}{2} I_{1} \otimes \rho_{2}-\frac{1}{2} \rho_{1} \otimes I_{2}\right)^{2} .
$$

After some algebra, this gives

$$
\operatorname{Tr} \rho^{\prime 2}=\operatorname{Tr} \rho^{2}-\frac{1}{2} \operatorname{Tr} \rho_{1}^{2}-\frac{1}{2} \operatorname{Tr} \rho_{2}^{2}+\frac{1}{2},
$$

which easily leads to the first Eq. (8).

Now we search for the density matrix $\rho^{\prime \prime}$, which makes $S_{12}^{\prime \prime}$ a maximum constrained by Eqs. (5). This puts forth a straightforward variational problem, and the solution is

$$
\rho^{\prime \prime}=\frac{1}{4}\left[I+\frac{1}{4} \beta B\right] .
$$

In fact, it is easy to prove that any density matrix fulfilling Eqs. (5) may be written in the form $\rho^{\prime \prime}+\delta \rho$ with $\rho^{\prime \prime}$ given by Eq. (11) and $\delta \rho$ Hermitian, traceless, and such that $\operatorname{Tr}(\delta \rho B)=0$. The entropy associated with such a density matrix fulfills

$$
S_{12}=S_{12}^{\prime \prime}-\operatorname{Tr}\left(\delta \rho^{2}\right) \leqslant S_{12}^{\prime \prime},
$$

the inequality coming from the property that indicates that the trace of the square of a Hermitian matrix is non-negative. The entropy $S_{12}^{\prime \prime}$ may be calculated by inserting Eq. (11) in to Eq. (2). We get

$$
S_{12}^{\prime \prime}=1-\frac{1}{16} \operatorname{Tr}\left[I+\frac{1}{2} \beta B+\frac{1}{16} \beta^{2} B^{2}\right]=\frac{1}{4}\left[3-\frac{1}{4} \beta^{2}\right],
$$

where we have taken into account the relations

$\operatorname{Tr} I=4, \quad \operatorname{Tr} B=0, \quad \operatorname{Tr} B^{2}=\operatorname{Tr}\left(4 I+\left[a_{1}, b_{1}\right]\left[a_{2}, b_{2}\right]\right)=16$.

Equation (6) follows immediately from Eqs. (8) and (13), if we take into account that $S_{12}^{\prime \prime}$, being a maximum, is not smaller than $S_{12}^{\prime}$. This completes the proof.

Our result include previous ones obtained within the information-theoretic approach, in particular the one obtained in Ref. [5], where the authors showed that from

$$
S(a, b) \geqslant \max \{S(a), S(b)\},
$$

the Bell-CHSH inequality can be derived, while to obtain the same result we have started from

$$
S(a, b) \geqslant \frac{1}{2}[S(a)+S(b)],
$$

It is easy to see that Eq. (15) implies Eq. (16), so our proof is less restrictive than theirs.

Some other interesting questions still open are whether a similar result could be obtained by using the standard von Neumann and Shannon entropies, instead of Eqs. (2), and if it would be possible to generalize these results to systems with more than two degrees of freedom. A particular example investigated in this respect [9] suggests that this would be the case.

We acknowledge financial support from DGICYT, Project No. PB-98-0191 (Spain).
[1] A. Wehrl, Rev. Mod. Phys. 50, 221 (1978).

[2] N. J. Cerf and C. Adami, Phys. Rev. A 55, 3371 (1997); Phys. Rev. Lett. 79, 5194 (1997).

[3] S. L. Braunstein and C. M. Caves, Phys. Rev. Lett. 61, 662 (1988).

[4] J. F. Clauser, M. A. Horne, A. Shimony, and R. A. Holt, Phys. Rev. Lett. 23, 880 (1969).

[5] R. Horodecki, P. Horodecki, and M. Horodecki, Phys. Lett. A 210, 377 (1996); R. Horodecki and M. Horodecki, Phys. Rev.
A 54, 1838 (1996).

[6] J. Von Neumann, Mathematical Foundations of Quantum Mechanics (Princeton University Press, Princeton, NJ, 1955).

[7] C. Tsallis, J. Stat. Phys. 52, 479 (1988); M. L. Lyra and C. Tsallis, Phys. Rev. Lett. 80, 53 (1998).

[8] S. L. Braunstein, A. Mann, and M. Revzen, Phys. Rev. Lett. 68, 3259 (1992).

[9] A. Mann, M. Revzen, and E. Santos, Phys. Lett. A 238, 85 (1998). 